(c)

(i) $\mathrm{BY}$

$\ominus$ ND

(5) $\mathrm{NC}$

\title{
EVALUACIÓN... MÁS DE LO MISMO DESAFIANDO FORMATOS Y MODALIDADES SIN LIBRETO
}

\author{
Evaluation... More of the same, challenging formats and modalities without script
}

Avaliação... Mais do mesmo desafiando formatos e modalidades sem roteiro

RECIBIDO:11DE ABRIL DE 2016

Daiana Yamila Rigo (Argentina)

daianarigo@hotmail.com

Universidad Nacional de Río Cuarto

Consejo Nacional de Investigaciones Científicas

y Técnicas

es

\section{RESUMEN}

El objetivo del trabajo es profundizar el planteamiento y la discusión sobre la necesidad de una evaluación más acorde con las nuevas concepciones del proceso de enseñanza y aprendizaje. El propósito general es describir las modalidades de evaluación más comunes en el aula, para generar propuestas educativas alternativas, delimitando criterios teóricos-prácticos para su formulación y puesta en acción. La investigación se realizó en el nivel primario de educación, junto a docentes y alumnos de sexto grado. Se diferenciaron dos etapas del estudio llevado a cabo. Primero, se observaron las prácticas más comunes y frecuentes de evaluación dentro de la asignatura de ciencias sociales, a modo de registrar su tipología y formato. Segundo, considerando la metodología estudio de diseño, se llevó a cabo una propuesta de evaluación, definida como contextualizada, auténtica y formativa. Para recolectar los datos se usó la observación y registro de grabaciones, e igualmente se administró un cuestionario con preguntas abiertas y cerradas a los estudiantes para rescatar su perspectiva sobre ambas modalidades de evaluación. Los resultados de la investigación muestran que la modalidad de evaluación propuesta ofreció un espacio para promover mejores aprendizajes en tanto es dinámica, progresiva y se encuentra ligada a un cambio en las prácticas de instrucción que soportan buenas instancias de feedback y la toma de decisiones por parte de los alumnos.

PALABRAS CLAVE: evaluación, instrucción, situaciones problemáticas, retroalimentación, estudios de diseño.

EVALUADO:19DE AGOSTODE 2016

ACEPTADO:30DE AGOSTO DE 2016

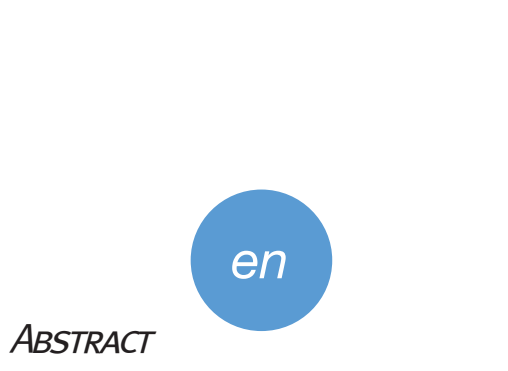

The purpose of this study is to deepen the approach and discussion about the need for an evaluation in line with the new conceptions of the teaching and learning processes. The general purpose is to describe the most common evaluation modalities in the classroom, to generate alternative educational proposals, delimiting theoretical and practical criteria for their formulation and implementation. The research was carried out at the elementary level of education, together with sixth grade students and teachers. Two stages of the study were differentiated. First, the most common and frequent evaluation practices within the social sciences subject were observed, in order to register their typology and format. Second, taking the design study methodology into account, an evaluation proposal was carried out, defined and contextualized, authentic and formative. To collect the data, recordings were observed and registered, and a questionnaire with open and closed questions was administered to the students to collect their perspective on both modalities of evaluation. The results of the research show that the proposed evaluation modality offered a space to promote better learning as it shows to be dynamic, progressive and linked to change in instructional practices that support good feedback and decision making by the students.

KEYWORDS: evaluation, instruction, problematic situations, feedback, design studies.
Danilo Donolo (Argentina) donolo@omail.com

Universidad Nacional de Río Cuarto Consejo Nacional de Investigaciones Científicas y Técnicas

PARA CITAR ESTE ARTÍCULO/TO CITE THIS ARTICLE/PARA CITAR ESTE ARTIGO:

Rigo, D. Y., \& Donolo, D., (2016). Evaluación... Más de lo mismo desafiando formatos y modalidades sin libreto, Panorama 10(19), p. 62-75. 
INTRODUCCIÓN

Pensar en evaluación es también considerar la nueva educación que deseamos. Nos parece interesante e ilustrativo, para iniciar la temática que deseamos estudiar una viñeta, a modo de disparar la reflexión, que encontramos hace algún tiempo en las redes sociales ${ }^{1}$. Se representa una situación de evaluación, en la cual el maestro les dice a sus alumnos, un grupo de animales: "Para que la evaluación sea justa, todos realizaréis la misma prueba: vais a subir a ese árbol".

Seguramente, usted ya habrá tenido contacto con la escena y la oportunidad de construir sus propias conclusiones sobre qué evaluamos y cómo lo hacemos. Creemos que las pruebas académicas no se distancian demasiado de la situación retratada, por el contrario, encontramos una cercanía curiosa, y preocupante a la vez, de concebir sobre todo la instrucción y su consecuente valoración como homogéneas y encapsuladas.

Por el contrario, los nuevos desarrollos teóricos nos motivan a transitar otros caminos alternativos para la evaluación que se asemejan al cuento narrado por Blasco (2008), sobre los desafíos del caracol y su rival, el rey. El autor muestra la necesidad de plantear desafíos justos a las habilidades de los estudiantes, que son diversas y distintas en términos de habilidades y competencias, tal cual lo plantea Gardner (1983), en sus desarrollos teóricos y prácticos sobre las múltiples inteligencias.

A continuación realizamos un breve recorrido teórico sobre la definición y características relevantes de la evaluación que proponemos y sobre feedback formativo, para luego mostrar una experiencia educativa con el objetivo de generar escenarios evaluativos formativos, estudios llevados a cabo junto a alumnos y docentes de nivel primario de educación.

\section{EVALUACIÓN... RETOS EN SU REDEFINICIÓN}

Replantear la educación supone, entre sus elementos fundamentales, repensar las prácticas de evaluación que se ponen en juego en el interior de los procesos de

1 De la cual desconocemos autoría. Imagen tomada de https://es.pinterest.com/ pin/5592908474448529865/ enseñanza-aprendizaje $e^{2}$. Los avances teóricos crecieron con un impacto importante en los últimos años, sin embargo, se advierte menos desarrollos prácticos que muestren ejemplos concretos sobre cómo hacer una evaluación para aprender, donde se presentan dilemas que son necesarios empezar a discutir y reflexionar.

$\mathrm{Al}$ respecto, Trillo Alonso (2005) reflexiona que saber evaluar se convierte en un asunto complejo y delicado, en tanto implica una serie de interrogantes que necesitan ir adoptando nuevos sentidos en la construcción dialéctica teórica-práctica, para qué, cómo, por qué, qué, a quién y quién evalúa. Lo cual exige una actuación profesional seria y fundamentada, reflexiva, deliberada, intencional, sistemática y, sobre todo, justificable.

En esta discusión, lo primero a reflexionar es la función de la evaluación y con ello delinear alguna clasificación. Podemos evaluar para examinar y calificar, y en eso estaríamos optando por una evaluación normativa, sumativa, que tiene una dirección clara hacia la valoración de los errores (Bahón, 2015). O bien, se puede optar evaluar para aprender, y se estaría eligiendo una modalidad que prioriza un proceso reflexivo, de toma de decisiones, que promueve el aprendizaje autónomo y autorregulado y sobre todo ayuda a redefinir la instrucción. Ciertamente, apostamos a esta última modalidad de evaluación, sin desconocer los compromisos que a veces nos atan a la acreditación del conocimiento a partir de una nota.

Pensar la evaluación es imprescindible, en tanto es considerada clave en el proceso de aprendizaje; al respecto, Boud (1998) considera que las modalidades y los requisitos de la evaluación posiblemente tienen más peso en cómo y qué aprenden los estudiantes, que cualquier otro factor individual. Incluso, es posible que tenga mayor importancia que el impacto de los materiales de la enseñanza.

Son muchos los investigadores que estudian y definen la evaluación desde una perspectiva innovadora. Alonso Tapia y de la Red Fadrique (2007) proponen

\footnotetext{
2 El tema de la formación de los niños y jóvenes y la necesidad de certificar la habilitación no es un tema menor en el mundo actual. Cada vez es más necesaria una capacitación más amplia y ajustada para atender a trabajos y profesiones que exigen saber lo básico y luego estar acorde con actualizaciones constantes. Un reciente estudio marca que las personas adultas son las que más padecen la desocupación, justamente por no estar actualizados. Y todavía es más difícil para ellos, porque ya han perdido el incentivo de buscar trabajo con las graves consecuencias e implicancias sociales que esto trae. Consultar: http://www.bbc.com/ mundo/noticias-36602737
}

Evaluación..

Más de lo mismo

desafiando

formatos y

modalidades sin

libreto . 
Daiana Yamila

Rigo I

Danilo Donolo I

Panorama I

pp. 62-75।

Volumen 101

Número 19 |

Julio-diciembre

2016 I integrar la evaluación al proceso de aprendizaje en lugar de hacerse básicamente al final, acompañada regularmente de retroalimentación sobre cómo superar las dificultades; todo ello en el contexto de un proceso orientado a la adquisición de competencias percibidas como relevantes, en donde el alumno tuviese la posibilidad de autorregular su aprendizaje en vez de tener que apoyarse en la regulación establecida por sus profesores. Para García-Jiménez (2015), lo que da sentido a la evaluación es la información que devuelve sobre el programa, la institución, la enseñanza o el aprendizaje y fundamentalmente que, a partir de ella, las personas implicadas pueden tomar decisiones sobre el mérito y el valor de lo evaluado o mejorar sus actuaciones. Bennett (2011) entiende que la evaluación no es un simple resultado, sino un proceso, del cual emerge una visión cualitativa de la comprensión del estudiante, utilizada para adaptar la enseñanza a las necesidades del alumno. Una adaptación que ocurre en ciclos cortos, dentro del aula y entre los implicados en la experiencia educativa. Rochera y Mauri Majós (1997) consideran a la evaluación, desde una perspectiva constructivista de orientación socio-cultural, como un instrumento fundamental para que el profesor pueda regular su acción docente a lo largo del proceso y para que el alumno pueda gestionar su propio proceso de aprendizaje.

Este enfoque muestra un giro respecto a la práctica sumativa de evaluación, revalorizándola como continua y en íntima conexión con el contexto instructivo -de contenidos, formatos, herramientas y representacionespara proporcionar información específica y para la toma de decisiones tendientes a mejorar la comprensión y competencias de los estudiantes y las enseñanzas.

Ravela (2009) menciona que las tareas que los maestros proponen a sus alumnos para evaluar el aprendizaje constituyen uno de los mejores indicadores del tipo de evaluación formulada, en tanto muestran qué es lo que los maestros valoran e indican a los estudiantes qué conocimientos y desempeños es importante alcanzar. $\mathrm{Si}$ las tareas requieren principalmente la memorización de conceptos y fechas, hacia allí estará orientado el esfuerzo de los estudiantes. En cambio, si las actividades implican desafíos para los alumnos, se encuentran contextualizadas, con sentido, e incitan a la reflexión, claramente motiva hacia otras metas de aprendizajes más complejas. Es decir, la evaluación condiciona la dinámica del aula, y los aprendizajes que allí ocurren.
Proponer un nuevo modelo de evaluación que se asocie a la idea de enseñar a los alumnos a pensar, decidir y actuar en el mundo real, supone conjugar tres conceptos centrales: auténtica, contextualizada y formativa. Herman, Aschbacher y Winters (1992) explican que demandar que los alumnos resuelvan activamente tareas complejas y auténticas mientras usan sus conocimientos previos, aprendizajes recientes y habilidades relevantes para la solución de problemas reales, habilita pasar de un modelo de evaluación descontextualizada, como lo son los exámenes escritos a final de tema, que evalúan un conocimiento declarativo, a una forma de evaluación contextualizada, para evaluar un conocimiento funcional (Trillo Alonso, 2005).

Una evaluación formativa, además de cumplir con los rasgos de situada y ligada a la vida cotidiana, debe proporcionar información a modo de generar retroalimentaciones e intercambios para modificar las actividades de enseñanza y aprendizaje en las que participan alumnos y docentes (Black y Wiliam, 1998). La clave, entonces, es encontrar, seleccionar y crear actividades que importen y que contribuyan a mejorar tanto la evaluación como el proceso de instrucción.

Bennett (2011), entiende que la evaluación formativa tiene como objetivo principal promover el aprendizaje, de modo tal, que cualquier modificación en el proceso instructivo esté asociado a una mejora educativa, atendiendo fundamentalmente a tres pilares: 1. Que los alumnos tengan en claro el objetivo de aprendizaje y de las tareas propuestas, 2. que los estudiantes tengan un espacio que soporte el monitoreo de su proceso de aprendizaje, y 3. que tomen decisiones orientadas a alcanzar sus metas (Osorio y López, 2014).

La evaluación para el aprendizaje tiene, además, como foco principal, la reflexión sobre lo que se va aprendiendo, en la confrontación entre lo que se intenta y lo que se alcanza, en la búsqueda de nuevos caminos para avanzar hacia los conocimientos y desempeños que se busca lograr (Ravela, 2009). Es un espacio que brinda información para comprender, conocer, interpretar y abrir distintos interrogantes, que permite no solo la 
corrección sino, fundamentalmente, mejorar tanto el aprendizaje como la enseñanza ${ }^{3}$.

En este modelo de evaluación, la idea de proceso cobra relevancia, en tanto se comprende que entre la evaluación, la enseñanza y el aprendizaje, existe una estrecha relación, tal que el profesor utiliza la evaluación como parte integral del proceso educacional para identificar dificultades y tomar decisiones tendientes a promover una mejora (Trumbull y Lash, 2013). La instrucción se entrelaza hasta que el proceso de evaluación en sí adquiere la forma de una nueva instrucción, en lugar de informar al estudiante únicamente sobre los errores o aciertos. Al respecto, Celman (2007), desde una perspectiva didáctica, y retomando los aportes de Litwin (1998), menciona que la evaluación significa también el estudio de las relaciones y las implicancias del enseñar $\mathrm{y}$ aprender.

La pregunta que surge en este contexto es sobre la posibilidad de hacer evaluaciones integradas al proceso de aprendizaje, y cómo concretarla en la práctica real del aula. Las actividades o situaciones de evaluación pueden ser de naturaleza muy diversa. Coll Salvador, Rochera Villach, Mayordomo Saíz y Naranjo Llanos (2007), proponen insertar las tareas de evaluación en las actividades de enseñanza-aprendizaje, organizar y secuenciar las actividades de evaluación en torno a bloques temáticos amplios, y facilitar al profesor el seguimiento, apoyo y tutorización de los alumnos durante el desarrollo de la misma.

Varios estudios encuentran que esta modalidad de evaluación ofrece un nuevo escenario para pensar las prácticas educativas. $\mathrm{Al}$ respecto, se remarca que las prácticas evaluativas constituyen momentos privilegiados del proceso de enseñanza-aprendizaje que los profesores pueden utilizar para ajustar mejor las ayudas que facilitan a los alumnos al hacer visibles los conocimientos que han construido en las situaciones de enseñanza-aprendizaje (Colomina y Rochera, 2002). Coll Salvador et al. (2007), además, resalta que las actividades de evaluación formativas son instrumentos útiles para obtener múltiples y diversas evidencias de los aprendizajes de los

3 Mucho tiene que ver aquí la formación de los docentes. El punto es también preocupación social. La referencia siguiente marca esa tendencia con notable claridad en los procedimientos seguidos por las instituciones para tener los mejores docentes, los más capacitados y los más actualizados. Consultar: http://www.bbc.com/mundo/noticias-36591625 estudiantes y proporcionan ayudas educativas ajustadas que favorecen el logro de aprendizajes significativos.

\section{FEEDBACK EN EL PROCESO DE EVALUACIÓN}

En todo proceso de enseñanza-aprendizaje se hace presente la retroalimentación entre los implicados. Su estudio ha evolucionado en la posibilidad de distinguir diferentes modalidades de generarlo y promoverlo. Los primeros estudios referían al feedback como una señal o información que se transmite de un emisor a un receptor; mientras que, actualmente, se lo caracteriza como un diálogo que entrelaza a profesores y alumnos en torno de etapas particulares en el desarrollo de tareas de aprendizaje. Al respecto, Rinaudo (2014) expone que entender la retroalimentación como un diálogo implica que el alumno no solo recibe información sobre su desempeño, sino que también tiene la posibilidad de participar en la reflexión del mismo.

Esta nueva perspectiva se retoma en la definición de evaluación formativa, pensada como un proceso cíclico que guía la instrucción y el aprendizaje, conteniendo al feedback como pieza fundamental y distintiva (Mandinach y Lash, 2016). Hattie y Timperley (2011) entienden que la retroalimentación se conceptualiza como la información proporcionada por un agente (maestro, compañero, libro, padre, alumno, entre otros), en relación con los aspectos de la actuación o comprensión que se tiene sobre el proceso de aprendizaje-enseñanza. Específicamente, por feedback formativo se comprende aquella información comunicada al alumno que está destinada a modificar su pensamiento o conducta con el fin de mejorar el aprendizaje. Del mismo modo, el maestro también puede recibir retroalimentación formativa y utilizarla como base para modificar la instrucción. La información que recibe el alumno para que la retroalimentación sea afectiva puede ser de dos tipos: de verificación -el simple juicio de si una respuesta es correcta- o de elaboración - mensaje que proporciona indicaciones pertinentes para guiar al estudiante hacia una respuesta correcta. Los investigadores parecen estar convergiendo hacia la opinión de que un feedback efectivo debe incluir ambos elementos (Shute, 2007).

Estos intercambios tienen el potencial para hacer explícito el pensamiento de los estudiantes, y por lo tanto, abierto a examen y revisión. Al respecto, Winne y Butler (1994) afirman que la retroalimentación es la
Evaluación..

Más de lo mismo

desafiando

formatos $y$

modalidades $\sin$

libreto
I Panorama

I pp. 62-75

I Volumen 10

I Número 19

I Julio-diciembre I 2016 
Daiana Yamila

Rigo I

Danilo Donolo I

\section{Danilo Donolo}

Wiggins (1998) puntualiza al respecto que la evaluación para mejorar el aprendizaje debería ofrecer retroalimentación de calidad a los estudiantes, no solo después de la actuación, sino también durante (concurrente con) las actividades de evaluación, como parte esencial de cualquier proceso de aprendizaje. Nótese, que este punto de vista desdibuja los límites entre la instrucción y la evaluación de una manera útil. En tanto se piensa al feedback no solo como valoraciones acerca del trabajo realizado o como orientaciones acerca de cómo mejorarlo, sino como devoluciones a lo largo del proceso de realización de las tareas, que le permiten al alumno comparar lo que intentó lograr con lo que efectivamente hizo. Cuanto más autoevidente sea la devolución, mejor será, porque ayudará al estudiante a darse cuenta por sí mismo de lo que ha logrado y lo que todavía no.

En este sentido, las prácticas evaluativas constituyen momentos privilegiados en que el profesor puede ayudar al alumno a hacer visibles los conocimientos construidos conjuntamente en el aula. Sin embargo, como advertimos, muchas veces las tareas utilizadas no permiten identificar el origen de las dificultades de los alumnos, razón por la que no se les puede ayudar a superar las dificultades mediante una retroalimentación precisa. Este escenario es particularmente preocupante cuando está demostrado que la retroalimentación frecuente y precisa es uno de los factores que más contribuye a la mejora de la motivación, el compromiso y los aprendizajes (Alonso Tapia, et al., 2007; Colomina, et al., 2002).

Panorama I

$4 \quad$ El tema de una formación de calidad desde los primeros grados de escolaridad tiene su correlato en la expectativa que la sociedad tiene sobre los jóvenes y su rol en la comunidad local y ampliada con referencia a las mismas futuras por las exigencias de la sociedad. En este sentido, estudios prospectivos marcan el camino de una mayor formación educacional y, sobre todo, universitaria para atender los requerimientos laborales y ocupacionales tanto independientes como asociados a empresas y grupos de trabajo. Los gráficos y las explicaciones del link que sigue es una tendencia posible. Consultar: http://www.lanacion.com. ar/1910320-el-futuro-de-la-educacion-mundial
Potencialidad que se consigue si la retroalimentación se da bajo determinadas condiciones, tales como ofrecerse en el momento adecuado durante el proceso de aprendizaje y no sólo al finalizar una tarea, y darse de manera precisa y con claridad para que los estudiantes puedan utilizarlo, significarlo y lograr progresos hacia las metas planteadas (Rochera Villach, Mauri Majós y Ginesta Fontseré, 2012). Igualmente, en un estudio de Alonso Tapia et al. (2007), se concluye que la evaluación de clase bastaría que se integrase en el proceso de aprendizaje en lugar de hacerse básicamente al final, seguida regularmente de retroalimentación sobre cómo superar las dificultades, para promover el aprendizaje.

En síntesis, proponer instancias de evaluación formativa tiene como finalidad generar cambios en las prácticas instructivas, y situar la valoración en un contexto educativo más amplio. Generar tareas académicas que, al mismo tiempo, sean actividades de evaluación que permitan al profesor recoger información sobre la comprensión de los contenidos alcanzados por los estudiantes y sobre la aplicación de estos para el desarrollo de la misma.

\section{ASPECTOS METODOLÓGICOS}

En la experiencia participaron estudiantes de sexto grado, de nivel primario de educación. En total fueron 24 alumnos y la docente de grado del área de ciencias sociales. La misma se desarrolló en el marco de una investigación de estudios de diseño (Rinaudo y Donolo, 2010), bajo el consentimiento informado de todos los implicados.

El objetivo del estudio fue describir y estudiar las prácticas de evaluación en el aula de nivel primario de educación y proponer alternativas de evaluación de carácter formativo y contextualizado. La investigación se desarrolló en dos etapas, una primera con la finalidad de describir las instancias de evaluación formuladas en el marco de la asignatura en estudio, realizando grabaciones y observaciones de clase, enfocadas a recabar información sobre la modalidad de evaluación -tipo de preguntas en tareas y pruebas escolares- $y$ las retroalimentaciones que se entablaban en ese espacio; y una segunda, orientada a desarrollar una experiencia de evaluación formativa, a fines de investigar espacios alternativos de evaluación como un proceso, con feedback orientados a facilitar y monitorear el aprendizaje de los estudiantes; usando 
como instrumentos de recolección de datos, grabaciones y observaciones no participantes de los momentos de retroalimentación entre alumnos-docentes-alumnos. Al concluir ambas etapas, se aplicó un cuestionario final corto con preguntas abiertas y cerradas para indagar la percepción que de esas prácticas los estudiantes tenían, a fines de rescatar fortalezas y debilidades, incluyendo preguntas para conocer la finalidad que cada modalidad de evaluación sostenía, ya sea orientada a la nota o a mejorar los aprendizajes, y para describir qué elementos fueron percibidos como positivos o negativos en cada instancia.

Con la información recolectada se llevó a cabo un análisis e interpretación del contenido de las retroalimentaciones, de las observaciones y registros obtenidos, a modo de comprender, por un lado, cada modalidad de evaluación y, por otro lado, las percepciones, valoraciones y aprendizajes vinculados a un formato de evaluación sumativa o formativa.

\section{DESCRIPCIÓN DE LA EXPERIENCLA 'EVALUACIÓN FORMATIVA'}

A modo de generar un contexto evaluativo formativo, considerando los desarrollos centrales que sustentan este tipo de prácticas, se diseñó una tarea académica que, en el marco de la asignatura funcionara como hilo conductor entre el proceso de aprendizaje, instructivo y evaluativo, con base en las interacciones entre docentes, alumnos y contenidos curriculares.

Específicamente, la propuesta de evaluación elaborada respondía en su formulación a la idea de contextualizada y a la vez ligadas a la vida cotidiana e integrada al proceso instructivo, con diversas instancias de feedback orientadas a favorecer mejores aprendizajes. El ejemplo que presentamos y analizamos, desde el estudio llevado a cabo, muestra un modelo de evaluación que denominamos Situación Problemática -SP-, una propuesta que rescata planteos desafiantes, pero a la vez interesantes y relacionados con la vida cotidiana, que no se resuelven en un hora, sino que implica un proceso que incluye y se lleva a cabo junto a las prácticas de instrucción. Son situaciones problemáticas, porque el estudiante necesita no solo de sus conocimientos previos para resolverla, sino que además se le presenta el desafío de buscar nueva información para completar la solución del problema formulado, que a la vez está asociado a circunstancias próximas de su vida fuera de la escuela. En la misma no se evalúa contenidos ni datos, sino más bien la comprensión de poner en juego los conceptos centrales del currículo de las ciencias sociales para analizar una situación cotidiana.

La situación problemática presentada consistía, grosso modo, en elegir algún lugar en el mundo explorando el globo terráqueo, con la finalidad de escribir una carta con destino a una adolescente que viajaría a ese mismo sitio. Los alumnos debían investigar y orientar a la joven viajera en el armado de la valija y ofrecer algunos tips para que no se perdiera y disfrutara de la cultura y hábitos del lugar. La tarea se desarrolló a lo largo de tres meses, mientras conjuntamente se iban debatiendo dudas que se respondían con nuevos contenidos o aprendizajes previos. Se previeron diversas instancias de feedback antes, durante y finalizada la tarea de evaluación y dos entregas preliminares, orientadas fundamentalmente a reflexionar sobre aspectos que ayudaran a gestionar $y$ monitorear la tarea para progresar en los aprendizaje, tales como: ¿cómo han encaminado la tarea y cuál es el objetivo que se propusieron?, ¿cuál es el estado de la tarea actualmente?, ¿cómo la están realizando?, ¿cuáles son y fueron las dificultades?, ¿cuáles serán los próximos paso a seguir?, entre otras. De la misma manera, los estudiantes junto a la docente discutieron aspectos esenciales para planificar y progresar en la SP: ¿qué les pide la tarea?, ¿cuál es el objetivo?, ¿qué tipo de información y estrategias necesitarán usar?, ¿cuánto tiempo y recursos necesitarán?, ¿comprenden lo que la tarea solicita?, ¿la tarea tiene sentido?, ¿están logrando el objetivo?, ¿necesitan cambiar algo?, ¿lograron el objetivo que se formularon?, ¿funcionó la modalidad de trabajo adoptada?, ¿cómo lo saben?, de lo contario, ¿qué podrían hacer para mejorar el trabajo la próxima vez?, ¿ cambiarían algo?, ¿qué?, las respuestas quedaron registradas por escrito en los cuadernos de los estudiantes.

Cabe destacar que los alumnos contaron con un cuaderno borrador para ir registrando los avances, las dudas, las consultas, las búsquedas realizadas y los avances preliminares de la escritura de la carta, como forma de ir consignando el proceso completo de investigación para resolver la SP.
| Evaluación...

Más de lo mismo

desafiando

formatos $y$

modalidades sin

libreto

. 
RESULTADOS

Daiana Yamila

Rigo I

Danilo Donolo I emáticas. Agrupando instrucción y evaluación; y Percepciones sobre las modalidades de evaluación.

ETAPA I- GUARDAR, COPLAR Y PEGAR... DEL LIBRO AL CUADERNO, DE LA CABEZA AL PAPEL

De las observaciones llevadas a cabo en la primera instancia de investigación, advertimos que la evaluación era más de lo mismo, es decir, preguntas con única respuesta, tal cual se formulan previamente en las tareas llevadas a cabo como estrategia principal de enseñanza y aprendizaje.

Primero, la acción de copiar y pegar va del libro de ciencias al cuaderno de clase, para luego realizar un registro más o menos fiel en la memoria para volcarlo al papel de evaluación. La concepción de evaluación que subyace a estas acciones es sumativa, en tanto es usada para emitir un juicio final sobre el aprendizaje de los estudiantes, al terminar un período de aprendizaje para evaluar el nivel de comprensión o competencia alcanzada (Jones, 2005).

Algunas de las preguntas registradas en esta instancia buscan que los estudiantes ofrezcan datos. Transcribimos a continuación algunos ejemplos de tareas de aprendizaje:

Copia

Trabajamos la pág. 262 del libro Biciencia

Leemos y contestamos

Panorama I

pp. 62-75।

Volumen 101

Número 19।

Julio-diciembre

2016 I

1. ¿Qué son las leyes?

2. ¿Qué es la Constitución Nacional?

3. ¿Cuándo se sancionó? ¿Cuál fue su última reforma?
Dictado. Trabajar las páginas 255, 256, 257, 258 y 259 del libro Biciencia

1. ¿Qué son los Derechos Humanos?

2. ¿Cuáles son sus características y cómo se los puede clasificar?

3. ¿Qué se entiende por minoría social o grupo vulnerable?, ¿cómo se intenta proteger los derechos de estos grupos?

4. ¿Qué hechos dieron origen a la DUDH?

5. ¿Cuándo fueron reconocidos constitucionalmente en Argentina los DDHH?

6. En la actualidad, ¿Qué personas y organizaciones luchan por la defensa de los DDHH?

Copia

Trabajamos con la Constitución Nacional

1. Leemos y analizamos algunos de los artículos que establecen los derechos de las personas y de los ciudadanos.

Artículos: 14,14 bis y 20 .

Artículo: 37.

Preguntas similares se encuentran en los registros de las evaluaciones sobre los conceptos trabajados, como se observa en los próximos extractos:

Respondan:

¿Qué es un derecho?, ¿Qué es la Constitución Nacional?

Lean y respondan verdadero o falso. Justifique las respuestas falsas.

- De acuerdo con el artículo 37 de la Constitución Argentina, el voto no es obligatorio.

- $\mathrm{El}$ artículo 14 bis de la Constitución Argentina establece los derechos del trabajador.

Con los siguientes conceptos elabore un texto:

Democracia-votos-derechos-autoridadeslibertad de expresión.

En ambas instancias, las preguntas de carácter factico son las que priman, donde fundamentalmente se resalta y valora un conocimiento que es más declarativo que funcional (Trillo Alonso, 2005), que motiva la memorización, más que la reflexión, el análisis y la comprensión. Furman (2015) aclara que el problema no está en este tipo de preguntas, sino que sean solo esas las presentes 
en los pizarrones. Al respecto, Jones (2005) indica que la evaluación sumativa puede y debe ser útil tanto como marco formativo y como parte del proceso de aprendizaje; la meta es lograr un entorno positivo de trabajo en torno a las respuestas, y no finalizar en una nota. Ir más allá de las respuestas cortas, sobre definiciones, fechas y datos, y apostar al análisis contextualizado, debate comprensivo y generación de opiniones.

Respecto a las retroalimentaciones, en la primera acción de copie y pegue se registraron algunos intercambios en torno a la tarea, como se puede leer en el siguiente extracto:

Revisamos, corregimos y completamos la tarea de la clase anterior

La maestra solicita los niños que se agrupen para evaluar los deberes.

M. ¿Cómo les fue con los deberes?

A1. Fácil, seño, estaba todo en el libro.

M. ¿Qué vimos la clase anterior?

A2. Las provincias estaban apartadas de Buenos Aires.

M. ¿Qué le reclamaban a la Provincia de Buenos Aires?

A3. El comercio.

M. Bien, ahora en grupo controlen la tarea.

Registro de observación: En el interior de los grupos, los estudiantes solo se limitan a leer las respuestas en función de las preguntas que tenían que contestar como deber; los que no leen no prestan atención a la respuesta que el compañero lee o siguen con la vista sus respuestas, no hay intercambios entre ellos, solo leen y si alguno dice que presenta una diferencia lee su respuesta. No se producen enriquecimientos mutuos o reformulación de las respuestas.
Del total, 6 alumnos no realizaron la tarea, o solo las marcaron en el libro de ciencias. En los grupos pasa la docente si los alumnos la llaman, si no, se mantiene alejada sin supervisar a los grupos que no demandan su consulta. Si hay alguna discordancia en las respuestas de un alumno y otro, la dejan, no llegan a un acuerdo y no reflexionan sobre las distintas respuestas escritas, buscando razones... dicen: "luego le preguntamos a la seño". $\mathrm{Si}$ a un alumno le falta una parte de la respuesta, otro se la dicta. Y pasan a la siguiente respuesta.

Como se puede observar, en el breve comentario de las retroalimentaciones en el interior del grupo, estas se limitan a una tarea de control, de completar y agregar información. No se motiva a argumentar o debatir más allá de las respuestas elaboradas.

Durante la segunda acción de guardar, para luego pegar en el papel, es decir, la instancia estrictamente de evaluación, las interacciones se redujeron a preguntas concretas para ampliar la comprensión sobre lo solicitado en cada pregunta, o confirmar lo que se creía que era correcto escribir, o bien consultas ajenas a la temática de evaluación, por ejemplo:

A1. Seño, acá tenemos que poner la definición de...

M. Sí.

A2. Puedo ir al baño,

M. No.

A3. Préstame la goma

A4. Acá tenés.

A5. Seño, ¿acá sería como vimos en clase?

M. Sí, María.

M. ¡Están muy perdidos, chicos!

A6. No me sale la $2 \mathrm{~b}$

M. ¿Qué dice el artículo 14 bis?

En este caso, coincidimos con Hattie y Timperley (2007), que la evaluación en la clase fomenta el aprendizaje superficial y rutinario, concentrándose en el recuerdo de detalles aislados, generalmente datos y definiciones que los alumnos olvidan pronto, que no requieren la mayoría de las veces grandes retroalimentaciones,
Evaluación..

Más de lo mismo

desafiando

formatos y

modalidades sin

libreto
I Panorama

I pp. 62-75

I Volumen 10

I Número 19

I Julio-diciembre I 2016 
Daiana Yamila

Rigo I

Danilo Donolo I

Danilo Donolo

En síntesis, en esta primera etapa se observa que instrucción y evaluación son dos instancias distintas, pero semejantes. Distintas porque ocurren en tiempos diferentes, primero se enseña y luego se evalúa lo aprendido; y semejantes, en cuando al tipo de preguntas que se formulan en uno y otro proceso. Respecto a la retroalimentación, observamos que se limitan a entregar información o a certificar un saber o respuesta. No se registraron instancias que inviten a repensar las respuestas o mejorar las producciones. Fueron respuestas cortas, donde predominaron los adverbios sí o no.

\section{Etapa II- Situaciones PROBLEMÁticas. Agrupando INSTRUCCIÓN Y EVALUACIÓN}

De los registros y análisis de la segunda etapa de investigación, encontramos que las preguntas elaboradas antes, durante y finalizada la SP, orientadas a planificar y progresar en su resolución, muestran que los estudiantes lograron formular metas claras de aprendizaje respecto de la tarea a desarrollar. Así mismo, en las respuestas ofrecidas se pone en evidencia un proceso de revisión y continuo monitoreo por parte de los estudiantes, facilitado tanto por el feedback externo recibido en las instancias de orientación y revisión del proceso, como por el feedback interno alcanzado por cada alumno al evaluar sus progresos (Rigo, en prensa). Como se pude leer en los siguientes extractos:

La tarea solicita que la ayudemos a planificar un viaje (TK).

La tarea pide que ayudemos a una chica y ser como una guía en el viaje (TC).

Panorama I

La tarea... ayudemos a una chica a armar su valija y escribirle una carta para enseñarle las costumbres, comidas típicas, idioma... (TL).
Escribir una carta con información del lugar, para que ella pueda realizar su viaje... orientarla en su viaje (TCS).

Aunque se nos presentaron varios obstáculos, los pudimos pasar a todos y lograr un buen trabajo (TLC).

El material de internet a veces era confuso, pero lo solucionamos porque fuimos a la biblioteca y encontramos la información clara (TKE).

Cambiaría la distribución del tiempo y trabajar más (TCP).

Necesito cambiar la forma de buscar información y conocer otras herramientas y recursos para acceder a nuevos conocimientos, saberes e información (TM).

Los estudiantes no solo explican cómo a lo largo del proceso van evaluando su nivel de comprensión, sus esfuerzos y estrategias usadas, sino también indican los cambios que planean realizar. En este contexto, el papel del docente en la evaluación formativa no es simplemente utilizar retroalimentaciones para promover el aprendizaje de contenidos, sino también ayudar a los estudiantes a comprender la meta, ayudarles a desarrollar las habilidades para hacer juicios sobre su aprendizaje y establecer un repertorio de estrategias para regular su propio aprendizaje (Heritage, 2010). Las ideas de Mandinach y Lash (2016), son propicias para entender que la evaluación formativa es una instancia que facilita la clarificación de los objetivos y metas de aprendizaje, promoviendo el conocimiento de estrategias y haciendo consciente las dificultades, los recursos a usar y la gestión del tiempo, tal como se observa a la largo del proceso de solución de la SP que los alumnos llevaron a cabo. Al respecto, Hattie y Timperley (2007) destacan el rol de las retroalimentaciones orientadas a promover procesos de autorregulación, remarcando la importancia que preguntas tales como ¿hacia dónde voy?, ¿cómo lo estoy haciendo?, ¿cuáles son los siguientes pasos a realizar? tienen para generar pensamientos, acciones y sentimientos que son planificados y adaptados cíclicamente para lograr las metas de aprendizaje formuladas (Zimmerman, 2000).

Igualmente, de las observaciones desarrolladas se registró que, a medida que los estudiantes avanzaban en la tarea de investigación, surgían preguntas que debían 
atenderse desde los aspectos conceptuales de la materia, entrelazando, en un ciclo iterativo, instrucción y evaluación. Entre esas preguntas surgieron:

¿Por qué en agosto en Europa es verano y en América del Sur es invierno?, ¿por qué hay diferencia horaria entre el lugar de salida y los diversos destinos propuestos para el viaje?, ¿por qué algunos lugares son cálidos o tropicales todo el año?, ¿`cómo se explica el tipo de clima de cada región?

Estos interrogantes abrieron un espacio instructivo orientado a nuevas explicaciones y exposiciones del docente hacia el logro de una mayor y mejor comprensión de la información encontrada y necesaria para continuar con el proceso de búsqueda y de recolección de datos para escribir la carta. Las preguntas formuladas por los estudiantes ofrecieron evidencias que, interpretadas y usadas por la docente, sirvieron para decidir qué pasos seguir en el proceso de aprendizaje, habilitó un ciclo formativo entre enseñanza y evaluación. En otras palabras, se redefinió el proceso instructivo en función de las retroalimentaciones que la maestra recibió de los alumnos. En este sentido, los contenidos no se ofrecieron de manera aislada mediante guías de preguntas, sino que surgieron de la interacción entre los alumnos frente a la resolución de la SP, como modalidad para ampliar y clarificar la comprensión. En este orden de ideas, la dirección del feedback no siempre es de docente a estudiante. Aquí, la evaluación formativa se comprende como uno de los instrumentos para informar y mejorar la planificación instruccional que realiza el docente (Heritage, 2010), caracterizada por ser progresiva y dinámica, donde no necesariamente primero se da la instrucción y luego la evaluación del estudiante, sino que se piensan como momentos en continuo movimiento de contracción y expansión conformando un ciclo iterativo (Mandinach y Lash, 2016).

De las grabaciones de las retroalimentaciones entre alumnos y docentes, se muestra a continuación algunos de los intercambios que tuvieron lugar en esta etapa de investigación:
M. ¿Cómo podrían hacer para seguir avanzando en la búsqueda de la información?

A1. Seguir buscando por internet. Pero para saber si el cambio de hora está bien... $\mathrm{mmmm}$.

A2. Podríamos preguntar en los foros, a esa gente que vos escribís una pregunta y luego te la contestan.

M. Me parece una muy buena alternativa. A3. Seño, la verdad que estoy atrasada, no busqué mucha información.

M. Isabela, ¿cuáles podrían ser las siguientes búsquedas a realizar?

A3. Pensé en ir a la biblioteca.

M. Bien, y ¿cómo organizarás la búsqueda?

A3. Voy a realizar como hice antes, una lista de preguntas para guiarme sobre lo que me falta, como hice la vez pasada, eso me ayudó.

M. Muy bien, Isabela, creo que podrás avanzar en la escritura de la carta.

M. ¿De qué modo podrían reformular el inicio de la carta? pensando en el destinatario, una adolescente de 15 años.

A4. Mmm sí, eso lo pensamos, no queda 'Estimada señora'.

A5. Tendría que ser menos formal.

A4. Algo como... 'Hola Constanza'.

M. Creo que podría ser una buena forma de reformular el saludo inicial.

Registro de observación: en el interior de los grupos, los estudiantes dispuestos en parejas organizaban previamente y registraban en un cuaderno borrador la información que necesitan para escribir la carta, algunos a través de preguntas - ¿cómo es el clima en Cuba en agosto?, ¿qué podría visitar la chica?, ¿cuál es el transporte que debe usar?, ¿qué ropa tiene que llevar?, ¿qué temporada es?- y otros a través de criterios o dimensiones, tales como costumbres, hábitos, lugares a visitar, tipo de cambio, comida, clima, fiestas, entre otras.

En la experiencia de evaluación formativa, se observa que los feedback que tuvieron lugar fueron más allá de resaltar los errores o simplemente corregir los deberes, es decir, no era una típica devolución orientada al resultado final, sino más bien como lo mencionan Ruiz-Primo y
Evaluación..

Más de lo mismo

desafiando

formatos y

modalidades sin

libreto
I Panorama

I pp. 62-75

I Volumen 10

I Número 19

| Julio-diciembre I 2016 
Daiana Yamila

Rigo I

Danilo Donolo I

En síntesis, instrucción y evaluación conformaron un ciclo que se enriqueció mutuamente, con la finalidad de monitorear, evaluar y mejorar la enseñanza y enriquecer el aprendizaje de los estudiantes. Del mismo modo, las instancias de retroalimentación orientadas a mejorar el aprendizaje proporcionaron al alumno sugerencias para promover el logro de la tarea y a los docentes evidencia para modificar la instrucción; adoptando los estudiantes una participación activa durante el proceso destinado a mejorar su aprendizaje.

\section{PERCEPCIONES SOBRE LAS MODALIDADES DE EVALUACIÓN}

De las respuestas ofrecidas por los estudiantes al cuestionario, registramos valoraciones diversas sobre las modalidades de evaluación en estudio. Sobre la primera etapa, acordaron en un $89 \%$ que se trataba de una instancia que tenía como finalidad aprobar la materia y obtener una nota, resaltando, sobre todo, el aprendizaje memorístico y las claves necesarias para obtener una buena nota: por un lado, tener la carpeta completa y, por otro lado, saber las respuestas de las preguntas que se habían ofrecido como instancia de deber. Igualmente, algunos alumnos comentaron que aprender siempre del libro es aburrido, sobre todo destacando el papel de las nuevas tecnologías en el proceso de aprendizaje de las nuevas generaciones. Entre las ventajas rescatadas está la facilidad de esta modalidad de evaluación, y entre las complejidades encontraron la dificultad de tener que saber las definiciones de memoria.

Sobre la segunda etapa, los alumnos vislumbraron más posibilidades de repensar sus producciones y aprendizajes, reconocieron que la evaluación se trasforma en una instancia que se ve favorecida por los intercambios con la docente a lo largo de su producción en un $92 \%$. Los estudiantes rescataron las instancias de feedback recibidas durante el desarrollo de la experiencia, el acompañamiento de la docente y las posibilidades de repensar sus producciones en búsqueda de mejores aprendizajes. Marcaron como positivo la libertad de definir qué estudiar y cómo mediante el uso de las nuevas tecno72 | logías, como la búsqueda por internet, combinada con metodologías más tradicionales, como las explicaciones del docente.

\section{CONSIDERACIONES FINALES}

El estudio presentado muestra dos análisis sobre modalidades distintas de evaluación. Primero, una instancia más tradicional de valorar los contendidos escolares, que apunta a la evaluación de lo aprendido, y una segunda instancia que muestra una alternativa promisoria de la evaluación que favorece el aprendizaje.

De la primera etapa, concluimos que la evaluación es una instancia final, que reproduce más o menos lo solicitado en las tareas académicas de copia, dictado, preguntas y respuestas, hace énfasis en el recuerdo de definiciones y datos precisos, con intercambios que se limitan a la verificación, chequear si está o no bien una determinada respuesta. De la segunda etapa, consideramos que la evaluación formativa habilita un espacio de elaboración, reflexión y retroalimención que mejora y facilita el aprendizaje, en tanto ofrece instancias para repensar la tarea y avanzar hacia mejores logros, conformando un ciclo junto a instrucción.

Los resultados de ambas instancias permiten orientar algunas apreciaciones sobre evaluación en educación. En primera instancia, la importancia de integrar instrucción y evaluación, aspectos remarcados por los estudiantes en sus percepciones sobre la propuesta diseñada. Es interesante también rescatar aquello que los motiva del proceso de cambio llevado adelante, al parecer, según los estudiantes, manifiestan que incursionar hacia nuevos recursos didácticos, puede ser una buena alternativa para generar mejores aprendizajes, por ejemplo, el uso de las nuevas tecnologías de la información pareciera ser algo que les interesa, como alternativa a los clásicos manuales. De la misma manera, rescatamos de sus apreciaciones respecto al valor que le otorgan al soporte que el docente brinda en diversas instancias de la evaluación formativa, como guía y por medio de sus exposiciones orales que ayudan a propiciar un mejor resultado en la tarea académica que se esté llevando a cabo. Otro aspecto a rescatar del estudio es la importancia que los estudiantes le atribuyen a poder repensar las tareas que se están realizando, es decir, pareciera que los aprendizajes acompañados de reflexiones son pensados como un desafío frente a las tareas que solicitan solo memorizar información. La misma relevancia le atribuyen al hecho 
de poder optar y tomar decisiones durante el proceso de aprendizaje, aspecto que remarcan como positivo, tanto en las elecciones que pudieron realizar acerca de qué investigar, como en la autonomía que se favoreció al generar un espacio para usar más de un soporte para avanzar y buscar información.

En segunda instancia, consideramos que la lógica de copiar y pegar es una instancia para enseñar y evaluar temáticas específicas de las asignaturas, pero su valor radica en el acompañamiento de una lógica de pensamiento productivo sobre lo que se escribe y se reproduce textualmente del libro al cuaderno. Los resultados muestran que propuestas como las llevadas a cabo, que se acompañan de instancias de feecback que orientan la reflexión y el monitoreo de las tareas por parte del alumno, son elementos clave para configurar contextos educativos tendientes a promover un pensamiento autorregulado. Acompañar el proceso de planificación, revisión y reflexión, genera intercambios entre docentes-alumnos y alumnos-alumnos que entendemos ayudan a generar una modalidad de trabajo que, como se aprecia en los resultados encontrados, muestran ser modelos para avanzar hacia una mayor control y gestión de los propios procesos cognitivos implicados en la tarea de aprender. En este sentido, la evaluación formativa habilita un espacio para la reflexión que se aleja de la reproducción casi textual y la memorización, en tanto y en cuanto se define como procesual y no situada en un tiempo y en un espacio limitado. Por el contrario, ofrece retroalimentación de calidad a los estudiantes, no solo después de la actuación, sino también durante el desarrollo de las actividades de evaluación, situándola en un contexto educativo más amplio.

Así mismo, rescatamos el valor de los estudios de diseño, como metodología que permite ir reformulando y ajustando las experiencias educativas, a modo de ir redefiniendo la investigación, la cual se ve enriquecida no solo por los aportes teóricos de la psicología educacional, sino también por los contextos naturales de enseñanza-aprendizaje donde tiene lugar el estudio en desarrollo.

En síntesis, los resultados permiten suponer que, cuando instrucción y evaluación van de la mano, no como instancias o etapas diferenciadas, se logra atender a una definición práctica de evaluación formativa, una evaluación para aprender, no de lo aprendido. Estos aspectos se recogen en la riqueza de las retroalimentaciones y en las percepciones que los estudiantes poseen sobre la experiencia desarrollada.

NOTA. Muchas veces los hechos que tienen más prensa son los que muestran los fracasos en las evaluaciones. Cada grupo de participantes en el proceso de enseñanza y aprendizaje - maestros, alumnos, padres, escuela- trata en esos casos de encontrar justificaciones o deficiencias en las actuaciones de los otros, siendo los alumnos los que generalmente son indicados como los más señalados por sus incorrecciones. Sin embargo, la experiencia reseñada muestra que los resultados son mejores si los interesados están comprometidos en la tarea, y eso es muy bueno...

Cuando a un alumno exitoso se le preguntó por el método que seguía para estudiar, dijo que no tenía ninguno. Y luego agregó: "Lo importante es saber organizarse y saber que cuando hay que estudiar, hay que estudiar"5.

Nosotros, en estas experiencias instruccionales integrándonos con maestros, alumnos, contenidos y contextos, circunstancias y teorías, intentamos que cada uno en su lugar juegue las mejores cartas por una mejor escuela para que los alumnos tengan una actitud positiva hacia el saber, para luego poder desempeñarse responsable y solidariamente en las comunidades cada vez más complejas en las que deberá actuar y mostrarse.

\section{REFERENCIAS}

1. Alonso, J. y de la Red, I. (2007). Evaluar "para” el aprendizaje, aprender para estar motivado: el orden de los factores sí afecta el producto. Revista Española de Orientación y Psicopedagogía, 18(2), 241-253.

2. Bahón, J. (2015). El sistema educativo actual no evalúa los aciertos de los alumnos, sino los fallos. Diario E1 Mundo. Recuperado de: http://www. elmundo.es/comunidad-valenciana/2015/11/26/ $5656128822601 \mathrm{~d} 73498 \mathrm{~b} 45 \mathrm{cc} . \mathrm{html}$

3. Bennett, R. (2011). Formative assessment: A critical review. Assessment in Education: Principles, Policy \& Practice, 18(1), 5-25.

Aquí la historia por el gusto de estudiar: http://www.huffingtonpost.es/2016/06/22/ alumno-selectividad_n_10609998.html
| Evaluación...

Más de lo mismo

desafiando

formatos $y$

modalidades sin

libreto 
4. Black, P.y Wiliam, D. (1998). Assessment and classroom learning. Assessment in Education: Principles, Policy E Practice, 18(1), 5-25.

Daiana Yamila

Rigo I

Danilo Donolo I

6. Boud, D. (1998). The challenge of problem-based learning. London: Kogan Page.

7. Celman, S. (2007). Evaluación de aprendizajes universitarios. Más allá de la acreditación. Colección de Cuadernos de actualización para pensar la Enseñanza Universitaria, Universidad Nacional de Río Cuarto, 2(11).

8. Coll, C., Rochera, M., Mayordomo, R. y Naranjo, M. (2007). Evaluación continua y ayuda al aprendizaje. Análisis de una experiencia de innovación en educación superior con el apoyo de las TIC. Revista Electrónica de Investigación Psicoeducativa, 5(13), 783-804.

9. Colomina, R. y Rochera, J. (2002). Evaluar para ajustar la ayuda educativa. Cuadernos de Pedagogía, 318, 56-62.

10. Furman, M. (2015). Preguntas para pensar. Conferencia TEDxRíodelaPlataED. Recuperado de: http:/www.tedxriodelaplata.org/videos/ preguntas-para-pensar

11. García-Jiménez, E. (2015). La evaluación del aprendizaje: de la retroalimentación a la autorregulación. El papel de las tecnologías RELIEVE. Revista Electrónica de Investigación y Evaluación Educativa, 21(2), 1-24. Recuperado de: http:// www.redalyc.org/pdf/916/91643847005.pdf

12. Gardner, H. (1983). Frames of mind: The theory of multiples intelligences. New York: Basic.

13. Hattie, J.y Timperley, E. (2007). The Power of Feedback. Review of Educational Research, 77(1), 81-112.

14. Hattie, J. y Timperley, H. (2011). The Power of Feedback. Review of Educational Research March, 77(1), 81-112.

Panorama I

pp. 62-75।

Volumen 10 I

Número 19

Julio-diciembre

2016 |

16. Herman, J., Aschbacher, P. y Winters, L. (1992). A practical guide to alternative assessment. Alexandria, VA: Association for Supervision and Curriculum Development. Recuperado de: http://files.eric.ed.gov/fulltext/ED352389.pdf
17. Jones, J. (2005). Developing effective formative assessment practices in the primary modern foreign language (MFL) classroom. Encuentro, 15. Recuperado de:

18. http://dspace.uah.es/dspace/bitstream/handle/10017/568/06_Jones. pdf? sequence $=18$ is Allowed $=y$

19. Mandinach, E.y Lash, A. (2016). Assessment illuminating pathways to learning. En Corno, L. y Anderman, E. (Eds.). Handbook of Educational Psychology (390-401). New York: Routledge Taylor \& Francis Group.

20. Osario, K. y López, A. (2014). La Retroalimentación Formativa en el Proceso de Enseñanza-Aprendizaje de Estudiantes en Edad Preescolar. Revista Iberoamericana de Evaluación Educativa, 7(1), 13-30.

21. Ravela, P. (2009). Consignas, devoluciones y calificaciones: los problemas de evaluación en las aulas de educación primaria en América Latina. Páginas de Educación, 2, 49-89, Montevideo, Universidad Católica del Uruguay. Recuperado de:

22. https://www.academia.edu/1414659/ CONSIGNAS_DEVOLUCIONES_Y CALIFICACIONES_LOS_PROBLEMAS_ DE_LA_EVALUACI\%C3\%93N_EN LAS̄_AŪLAS_DE_EDUCACI\%C3\%93N_ PRIMARIA_EN_AM\%C3\%89RICA_

23. Rigo, D. (2016, en prensa). Autorregulación y rúbricas como herramienta de evaluación. Experiencia desarrollada en educación primaria. Revista Escuela Abierta, 19.

24. Rinaudo, C.y Donolo, D. (2010). Estudios de diseño. Una alternativa promisoria en la investigación educativa. RED - Revista de Educación a Distancia, 22. Recuperado de: http://www.um.es/ $\mathrm{ead} / \mathrm{red} / 22$

25. Rinaudo, M. (2014). Estudios sobre los contextos de aprendizaje: arenas y fronteras. En Paoloni, P., Rinaudo, M. y A. González. Cuestiones en Psicología Educacional. Perspectivas teóricas y metodológicas orientadas a la mejora de la práctica educativa (163-206). La Laguna: Sociedad Latina de Comunicación Social. Recuperado de: http://www.cuadernosartesanos.org/educacion. $\underline{\mathrm{html}}$

26. Rochera, M., Mauri, T. y Ginesta, A. (2012). Feedback participativo en la evaluación continuada. Ponencia presentada en el Congreso Internacional de Docencia Universitaria,

Barcelona, España. Recuperado de: http://www. cidui.org/revista-cidui12/index.php/cidui/ article/view/21/14

27. Rochera, M.. y Mauri, T. (1997). Aprender a regular el propio aprendizaje. Aula de Innovación Educativa, 67, 48-52. 
28. Ruiz-Primo, M. y Li, M. (2013). Examining

formative feedback in the classroom context:

New research perspectives. En J. M. McMillan

(Ed.). Handbook of research on classroom assessment

(215-232). Thousand Oaks, CA: Sage.

Evaluación.

Más de lo mismo

desafiando

formatos y

modalidades sin

libreto

30. Trillo, F. (2005). Competencias docentes y Evaluación auténtica: ¿Falla el protagonista? Colección de Cuadernillos de actualización para pensar la Enseñanza Universitaria. Universidad Nacional de Río Cuarto. Recuperado de: https:// www.unrc.edu.ar/unrc/academica/pdf/cuadernillo03.pdf

31. Trumbull, E., y Lash, A. (2013). Understanding formative assessment: Insights from learning theory and measurement theory. San Francisco: WestEd.

32. Wiggins, G. P. (1998). Educative assessment: designing assessments to inform and improve student performance. San Francisco: Jossey-Bass.

33. Winne, P. y Butler, D. (1994). Student cognition in learning from teaching. En T. Husen y T.

Postlewaite (Eds.). International encyclopaedia of education (pp. 5738-5745). Oxford, UK:

Pergamon.

34. Zimmerman, B. J. (2000). Attaining self-regulation: A social cognitive perspective. En M.

Boekaerts, P. R. Pintrich y M. Zeidner (Eds.).

Handbook of self-regulation (13-40). San Diego,

California: Academic Press. 